\title{
Clinical Importance of a Peritoneal Interposition Flap to Prevent Symptomatic Lymphoceles after Robot-Assisted Radical Prostatectomy and Pelvic Lymph Node Dissection: A Systematic Review and Meta-Analysis
}

\author{
Sebastian Deutsch ${ }^{a}$ Boris Hadaschik ${ }^{a, b}$ Steffen Lebentrau ${ }^{c}$ Burkhard Ubrig ${ }^{d}$ \\ Max Burgere Matthias May $^{f}$ \\ ${ }^{a}$ Department of Urology, University Hospital Essen, Essen, Germany; ${ }^{b}$ German Cancer Consortium (DKTK), University \\ Hospital Essen, Essen, Germany; 'Department of Urology, Brandenburg Medical School Theodor Fontane, Ruppiner \\ Kliniken, Neuruppin, Germany; ${ }^{d}$ Department of Urology, Augusta Kliniken Bochum Hattingen, Bochum, Germany; \\ eDepartment of Urology, Caritas St. Josef Medical Centre, University of Regensburg, Regensburg, Germany; \\ fDepartment of Urology, St. Elisabeth-Hospital Straubing, Brothers of Mercy Hospital, Straubing, Germany
}

\section{Keywords}

Peritoneal rotation flap - Peritoneal interpolated flap . Peritoneal Re-approximation - Pelvic lymphadenectomy . Meta-analysis · Systematic review

\section{Abstract \\ Background: Robot-assisted radical prostatectomy (RARP) including pelvic lymph node dissection (PLND) is the current state of the art in surgical therapy of localized prostate can- cer with intermediate or high risk. PLND in particular is asso- ciated with morbidity inherent to this method; the rate of symptomatic lymphoceles (sLCs), for example, ranges up to $10 \%$. Objective: Various intraoperative modifications have been developed with the aim of reducing the $\mathrm{SLC}$ rate. Based on current studies, a peritoneal interposition flap (PIF) ap- pears to be one of the most effective methods for this pur- pose. Under the criteria of a systematic review, 5 retrospec- tive studies have been identified until now, 4 of which showed a positive effect of PIF on the sLC rate. Results and Limitations: A total of 1,308 patients were included in the aggregated analysis of these 5 studies. The amount of sLCs}

was $1.3 \%(8 / 604)$ and $5.7 \%(40 / 704)$ in the PIF and standard groups, respectively $(p<0.001)$. The resulting odds ratio (OR) was 0.23 (95\% confidence interval [Cl]: 0.05-0.99), taking into account a noteworthy heterogeneity of the 5 studies $(Q=$ 9.47, $p=0.05 ; l^{2}=58 \%$ ). In addition, a prospective randomized and blinded study (Pianoforte trial) with corresponding sLC rates of $8.3 \%(9 / 108)$ versus $9.7 \%(12 / 124)(p=0.820)$ exists. In this study, the OR was 0.85 (95\% Cl: $0.34-2.10, p=$ $0.722)$. Conclusion: Despite positive results from retrospective studies with indirect evidence, the role of the PIF in the reduction of $S L C$ in RARP could not be conclusively assessed yet. The results of the first prospective randomized study do not show a positive effect of PIF, declaring a research gap for further studies with direct evidence.

(c) 2021 S. Karger AG, Basel

\section{Introduction}

In countries with availability and access, robot-assisted radical prostatectomy (RARP) is the gold standard surgical approach for localized prostate cancer karger@karger.com www.karger.com/uin

Karger"
(C) 2021 S. Karger AG, Basel

University Hospital Essen

Hufelandstr. 55, DE-45147 Essen (Germany)

deutsch.bastian@web.de 
due to fair oncological and functional outcomes and favorable morbidity $[1,2]$. Pelvic lymph node dissection (PLND) is a recommended part of the procedure in intermediate or high-risk cancer but, at the same time, a major cause of postoperative morbidity [3]. The rate of symptomatic lymphoceles (sLCs) ranges from 2 to $10 \%$ [4-10]. Therefore, various intraoperative modifications have been examined with regard to their impact on prevention or reduction of sLCs. Many initially promising results were not confirmed in validation studies, and research for the best approach preventing sLCs continues [10]. In 2015, Lebeis et al. [2] introduced the peritoneal interposition flap (PIF) as a simple intraoperative modification to reduce sLCs in RARP patients. Based on subsequent studies, PIF currently appears to be the most promising approach for the reduction or prevention of sLCs after robotic PLND. However, the evidence in support of PIF has been based on retrospective studies [4-8]. Only one multicenter randomized patient- and investigator-blinded trial of PIF versus no PIF in RARP patients has been published so far [9]. An aggregated analysis of the available studies for a better evaluation of PIF is currently lacking [11]. Therefore, further studies on PIF have been looked for, and a metaanalysis of sLC rates after PLND within RARP between PIF and no PIF was conducted.

\section{Methods}

\section{Search Strategy and Data Extraction}

This study was performed in accordance with the Preferred Reporting Items for Systematic Reviews and Meta-Analyses (PRISMA) guidelines [12]. PubMed, Embase, and Cochrane databases for published trials up to August 2020 have been searched. The following MeSH and main keywords were used: "prostate cancer," "robot-assisted radical prostatectomy" (or) "laparoscopic radical prostatectomy," "pelvic lymph node dissection," "symptomatic lymphoceles," "peritoneal flap" (or) "peritoneal interposition flap" (or) "P.L.E.A.T.," and associated terms. The language was not restricted. Also manual searches of the reference lists in the selected studies to retrieve all relevant data have been implemented. The inclusion criteria of the meta-analysis were defined as follows: retrospective or prospective studies on RARP (or laparoscopic) due to localized prostate cancer, in which the influence of a PIF on the formation of sLCs was analyzed comparatively. Two authors (S.D. and M.M.) independently assessed the identified studies and reviewed the abstracts to select full articles. The joint integration of prospective and retrospective studies within a meta-analysis was only favored if the methodological quality of the retrospective studies fulfilled the critical evaluation. Discussion was performed among all authors to resolve any disagreements. The study endpoint of sLCs was identical in the selected studies ([fever as a symptom of an infected lymphocele, lower abdominal pain, lower extremity swelling, and/or deep vein thrombosis] in combination with a pelvic fluid formation).

\section{Statistical Analysis}

The primary endpoint of this meta-analysis was the sLC rate and assessed using odds ratios (ORs). Where clinically appropriate, a meta-analysis was performed using a random-effect model due to heterogeneity in study design, intervention schedule, outcome definition, and time point or modality of measurement. ORs are presented with $95 \%$ confidence intervals (CIs). Cochran's $Q$ test and the $I^{2}$ statistic were used to evaluate the heterogeneity among the studies, and a $p<0.1$ was considered as statistically significant. For the meta-analysis, Review Manager Software (RevMan v.5.4; Cochrane Collaboration, Oxford, UK) was used to analyze the data. The risk of bias for the retrospective studies underlying the meta-analysis was assessed according to Cochrane guidelines [13]. Extra domains were used to assess confounders in nonrandomized studies: a pragmatic approach informed by the methodological literature [13]. It was assessed whether each prognostic confounder was considered, whether the confounder was balanced between the intervention and the control group, and whether, if necessary, the confounder was controlled for in the analysis. A list of the most important potential confounders for harm and benefit outcomes was developed a priori with clinical content experts of the work group: LN count, BMI, clip or coagulation of the lymphatic vessels, and the surgical expertise.

\section{Results}

All 6 included studies were retrieved using the search strategy according to the PRISMA guidelines (shown in Fig. 1). Due to significant qualitative differences in terms of standardization, timing, and also the extent of the follow-up for the specified endpoint, the present 5 retrospective studies were not included in a meta-analysis together with the prospective study. Finally, 5 retrospective comparative studies, which met the study inclusion criteria, were selected for meta-analysis (shown in Fig. 1; shown in Table 1) [4-8]. A total of 1,308 patients were included in the aggregated analysis of these 5 studies. The sLC rates account for $1.3 \%(8 / 604)$ and $5.7 \%(40 / 704)$ in the PIF and standard group, respectively $(p<0.001$; shown in Table 1). The resulting OR was 0.23 (95\% CI: $0.05-0.99)$, taking into account the heterogeneity of these studies $\left(Q=9.47, p=0.05 ; I^{2}=58 \%\right.$; shown in Fig. 2). In addition, a prospective randomized and blinded study (Pianoforte trial) with corresponding sLC rates of $8.3 \%$ $(9 / 108)$ versus $9.7 \%(12 / 124)(p=0.820)$ exists, and its results were compared to those of our meta-analysis (shown in Table 1) [7]. In this prospective study, the OR was 0.85 (95\% CI: 0.34-2.10). The risk of bias assessment of included studies is summarized in Table 2. 
Fig. 1. PRISMA flow diagram of studies identified, excluded and included. PRISMA, Preferred Reporting Items for Systematic Reviews and Meta-Analyses; PIF, peritoneal interposition flap; sCL, symptomatic lymphocele.

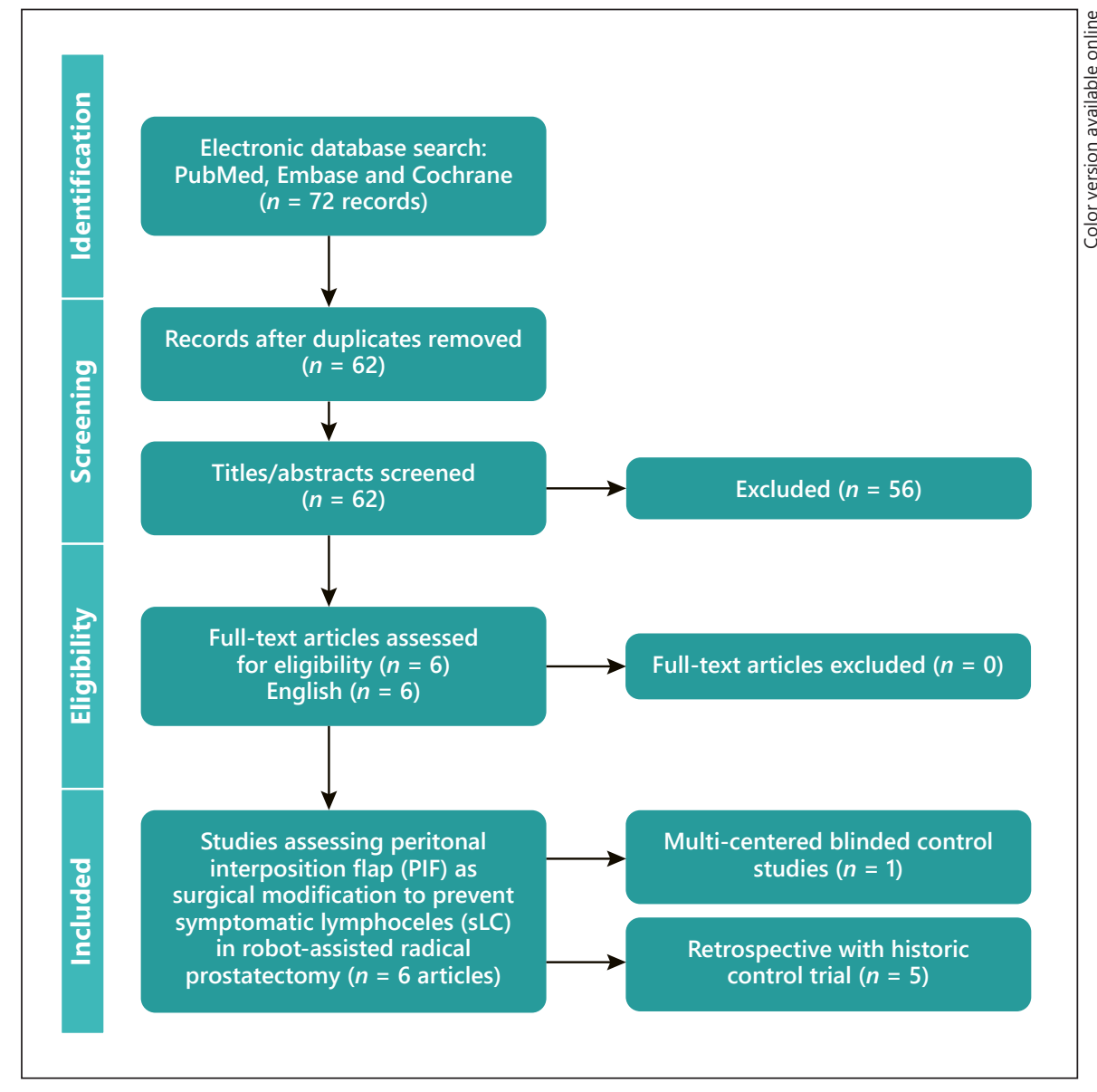

Table 1. Rates of sLCs in PIF and standard (no-PIF) groups in the included studies

\begin{tabular}{lccrr}
\hline Study & $\begin{array}{c}\text { \% sLC [PIF] } \\
\text { (event/total pts) }\end{array}$ & $\begin{array}{c}\text { \% sLC [no-PIF] } \\
\text { (event/total pts) }\end{array}$ & $p\left(\chi^{2}\right)^{*}$ & $p\left(\chi^{2}\right)^{* *}$ \\
\hline Lebeis et al. [4] & $0 \%(0 / 77)$ & $11.7 \%(9 / 77)$ & 0.002 & 0.003 \\
Dal Moro and Zattoni [5] & $0.6 \%(1 / 176)$ & $4.1 \%(8 / 195)$ & 0.027 & 0.039 \\
Stolzenburg et al. [6] & $1.0 \%(2 / 193)$ & $4.7 \%(9 / 193)$ & 0.031 & 0.062 \\
Boğa et al. [7] & $12.2 \%(5 / 41)$ & $5.3 \%(2 / 38)$ & 0.248 & 0.434 \\
Lee et al. [8] & $0 \%(0 / 117)$ & $6.0 \%(12 / 201)$ & 0.004 & 0.005 \\
Aggregated [4-8] & $\mathbf{1 . 3 \% ( 8 / 6 0 4 )}$ & $\mathbf{5 . 7 \% ( 4 0 / 7 0 4 )}$ & $<\mathbf{0 . 0 0 1}$ & $<\mathbf{0 . 0 0 1}$ \\
Bründl et al. [9] & $8.3 \%(9 / 108)$ & $9.7 \%(12 / 124)$ & 0.452 & 0.820 \\
\hline
\end{tabular}

PIF, peritoneal interposition flap; pts., patients; sLC, symptomatic lymphocele. * One-sided. ** Two-sided.

\section{Discussion}

As a result of the meta-analysis of the 5 available retrospective studies on the effect of PIF, a 77\% (95\% CI: $1-99 \%)$ reduction in the sLC rate was observed [4-8]. The certainty of this statement is limited by a considerable heterogeneity $\left(I^{2}=58 \%\right)$ and also by notable methodological differences between the retrospective studies (shown in Fig. 2; shown in Tables 2, 3).

The hoped for mode of action of PIF formation to reduce or prevent sLCs is the same in all studies. PIF is intended to ensure free drainage of lymphatic fluids out of 


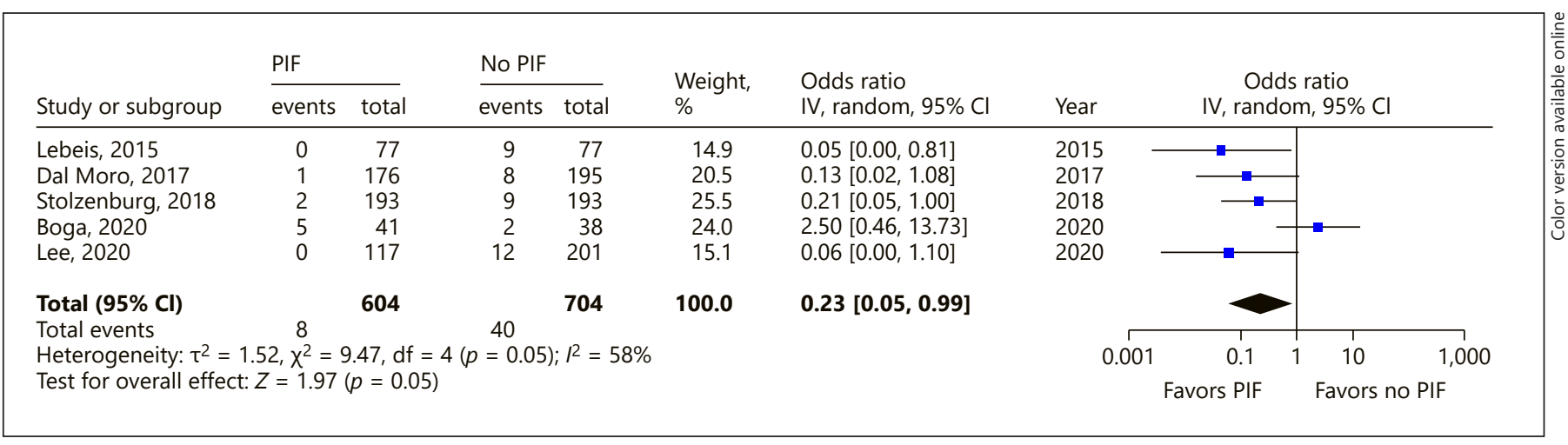

Fig. 2. Forest plot from the results of the 5 retrospective studies available assessing cumulative incidence of the endpoint sLCs after RARP and PLND in the groups PIF versus standard (no PIF) [4-8]. sLC, symptomatic lymphocele; PIF, peritoneal interposition flap; RARP, robot-assisted radical prostatectomy; PLND, pelvic lymph node dissection; OR, odds ratio; $\mathrm{CI}$, confidence interval.

Table 2. Risk of bias for included retrospective studies and the one prospective-randomized study

\begin{tabular}{lllllll}
\hline Study & $\begin{array}{l}\text { Allocation } \\
\text { concealment } \\
\text { (selection bias) }\end{array}$ & $\begin{array}{l}\text { Blinding of } \\
\text { participants } \\
\text { and personnel } \\
\text { (performance bias) }\end{array}$ & $\begin{array}{l}\text { Blinding of } \\
\text { outcome } \\
\text { assessment } \\
\text { (detection bias) }\end{array}$ & $\begin{array}{l}\text { Incomplete } \\
\text { outcome } \\
\text { data (attrition } \\
\text { bias) }\end{array}$ & $\begin{array}{l}\text { Selective } \\
\text { reporting } \\
\text { (reporting } \\
\text { bias) }\end{array}$ & $\begin{array}{l}\text { Other } \\
\text { bias }\end{array}$ \\
\hline Lebeis et al. [4] & $+/-$ & - & $+/-$ & $+/-$ & $+/-$ & $+/-$ \\
Dal Moro and Zattoni [5] & $+/-$ & - & $+/-$ & $+/-$ & $+/-$ & - \\
Stolzenburg et al. [6] & $+/-$ & $+/-$ & + & + & $+/-$ & + \\
Boğa et al. [7] & - & - & $+/-$ & $+/-$ & $+/-$ & $+/-$ \\
Lee et al. [8] & $+/-$ & - & $+/-$ & $+/-$ & $+/-$ & $+/-$ \\
Bründl et al. [9] & + & + & + & + & + & +
\end{tabular}

Boxes without a risk of bias assessment summary icons indicate studies from which data for this outcome category were unavailable. The evaluation of the column "other bias" was based on the balance of the following criteria in the group arms of the respective study: lymph node count, BMI, clip or coagulation of the lymphatic vessels, and the surgical expertise. +, low risk of bias; +/-, unclear; -, high risk of bias.

the pelvis into the abdominal cavity and to increase the peritoneal reabsorption area. The PIF principle was presented first in 2015 by Lebeis et al. [4], who compared 77 patients with this intraoperative modification (in the form of a 2-point fixation of the bladder peritoneum) to 77 historical patients without PIF and controlled the endpoint over a period of slightly more than 1 year (unicentric, 2 surgeons).

In 2017, Dal Moro and Zattoni [5] presented the results of a unicentric single-surgeon series in which 176 PIF patients and 195 historical standard procedures were compared (follow-up was 2 and $>5$ years, respectively). The PIF was performed in a surgical modification, with the adaptation of the bladder peritoneum being more in the midline (P.L.E.A.T.).
In their unicentric multiple-surgeon study, Stolzenburg et al. [6] performed a propensity score matching of 232 PIF patients with historical patients, resulting in a comparison group of 193 patients each. Besides the propensity score matching, a strength of this study is that the patients were examined in a structured manner on the ninetieth postoperative day. Differences compared to the initial description were that Stolzenburg et al. [6] performed a 4-point fixation of the bladder peritoneum. It should also be noted that only in the study by Stolzenburg et al. [6], the proportion of laparoscopic procedures is about $27 \%$ ( $73 \%$ of patients received RARP). All other available studies on the PIF considered only RARP procedures. 
Table 3. Number of surgically removed lymph nodes and distribution of BMI in the included studies

\begin{tabular}{|c|c|c|c|c|c|c|}
\hline Study & $\begin{array}{l}\text { LN count (median } \\
\text { with } \mathrm{IQR} \text { or } \\
\text { mean } \pm \mathrm{SD} \text { ) }[\mathrm{PIF}]\end{array}$ & $\begin{array}{l}\text { LN count (median } \\
\text { with IQR or } \\
\text { mean } \pm \text { SD) [no PIF] }\end{array}$ & $p$ value & $\begin{array}{l}\mathrm{BMI}, \mathrm{kg} / \mathrm{m}^{2}(\text { median } \\
\text { with IQR or mean } \pm \mathrm{SD}) \\
{[\mathrm{PIF}]}\end{array}$ & $\begin{array}{l}\mathrm{BMI}, \mathrm{kg} / \mathrm{m}^{2}(\text { median } \\
\text { with } \mathrm{IQR} \text { or mean } \pm \mathrm{SD}) \\
{[\text { no PIF] }}\end{array}$ & $p$ value \\
\hline Dal Moro and Zattoni [5] & $10(6.5-15)$ & $5(0-11)$ & $<0.001$ & na & na & na \\
\hline Stolzenburg et al. [6] & 15 (range: $5-33$ ) & 15 (range: 6-32) & 0.908 & 26.9 (range: $20.8-42.3$ ) & 27.2 (range: $19.9-41.8$ ) & 0.815 \\
\hline Boğa et al. [7] & $15.2 \pm 7.9$ & $19 \pm 10.7$ & 0.070 & $26.4 \pm 7.4$ & $27.4 \pm 5.0$ & 0.290 \\
\hline
\end{tabular}

IQR, interquartile range; LN, lymph nodes; na, not available; PIF, peritoneal interposition flap; SD, standard deviation.

In their unicentric study, Boğa et al. [7] evaluated 41 (PIF) versus 38 (no-PIF) patients who received RARP from a different surgeon in each group. Thus, their results not only reflect the comparison of methods but also the differences between the 2 surgeons. This Turkish study was largely responsible for the heterogeneity of the studies in the meta-analysis. If the results of this study are omitted from our meta-analysis, the heterogeneity of the remaining 4 retrospective studies will be $I^{2}=0 \%$, with an OR of 0.13 (95\% CI: 0.05-0.38). The peritoneal patch was realized by Boğa et al. [7] as a continuous ventral suture, and patients were surveyed with a mean postoperative follow-up of 19 months.

In their unicentric single-surgeon series, Lee et al. [8] compared 117 patients with PIF and 201 historical patients without PIF, with a mean follow-up of 7 and 14.3 months, respectively. The surgical procedure for the formation of PIF was similar to the initial description by Lebeis et al. [4, 8].

In addition to these 5 retrospective studies with only indirect evidence, there is one prospective randomized, patient- and investigator-blinded study (Pianoforte trial) to evaluate the effect of PIF on the sLC rate after RARP [9]. Since the Pianoforte trial was designed as a prospective validation of the Lebeis study, the effect size reported in the Lebeis study (Phi and Cramers V, each $=0.249$ ) was used for biometric sample size estimation [9]. According to the study protocol, sLCs at 2 different points of time (these are time at discharge [T1] and 90 days after surgery [T2]) were treated as co-primary endpoints; therefore, considering the multiple testing issue, a two-sided alpha error of $2.5 \%$ ( $p=0.025)$ was set for both endpoints. The type II error was set to $ß=0.2$ (power $80 \%$ ). The Pianoforte trial was designed with 1:1 randomization. Considering the parameters relevant for biometric planning, 77 patients per group were required. In order to increase the validity of the study results, the Pianoforte group intended to exceed the biometrically required sample size by fully using the planned study duration of 9 months, given the high level of patients' safety reported in the previous publication, resulting from the modification of the surgical technique. In the end, 108 (PIF) and 124 (no-PIF) patients were included in this study. The results of the multicenter Pianoforte trial with direct evidence do not show significant group differences in the sLC rate within a time window of 90 days postoperatively. Furthermore, prospective monitoring of study patients led to detection of more sLCs than those in the retrospective cohorts. In the Pianoforte trial, PIF was performed in analogy to the initial description as a 2-point fixation. Common to all 6 studies is that there was no increased complication rate or altered functional outcome in patients with PIF [4-9].

To ensure the comparability of the study arms of the 6 analyses, the balance of risk factors associated with the occurrence of sLCs has been assessed. The number of removed lymph nodes plays a decisive role in the development of lymphoceles [14]. In this respect, the study arms in the different studies are comparable (shown in Table 3). Overall, the low number of removed lymph nodes in the study by Lebeis et al. [4] is surprising. Another important criterion is the way in which the lymphatic vessels are treated in the context of PLND (coagulation or clipping) [15]. Unfortunately, only results from the prospective series are available, in which the proportion of patients with clipped lymphatic vessels was comparable $(60.2 \%$ in the PIF group vs. $67.7 \%$ in the no-PIF group, $p=0.272$ ) [9]. Interestingly, according to the results of recent studies, the postoperative development of sLCs seems to depend on the patient's BMI (higher BMI leads to more frequent sLCs) $[16,17]$. In this criterion, the group arms of the analyzed studies seem 
matchable (shown in Table 3). However, Dal Moro and Zattoni [5] did not state the BMI of their patients.

Another critical issue is to answer the question whether the follow-up underlying the studies in our meta-analysis is sufficient to robustly verify the sLC endpoint. The studies included in the meta-analysis showed a postoperative follow-up between 90 days and 5 years [4-8]. In a study by Andrews et al. [18] of more than 8,000 patients with an exceptionally long follow-up, the median time to develop sLCs was between 22 and 63 days. Although late sLCs may occur in isolated cases, this period coincides with the time intervals for the development of sLCs reported in other studies with a long follow-up. Therefore, a postoperative time interval of 90 days may be considered sufficient for the endpoint of sLCs $[4,5,7,8,10$, 14-17]. Another point that is often discussed in association with sLCs is the type of thromboembolic prophylaxis [19]. Although there were no group differences in the respective studies in this meta-analysis, there were differences in the type of heparin used (unfractionated or fractionated) and the length of heparin application in the comparison of the studies. However, all patients received daily heparin in a standardized dosage that was injected s.c. on the upper arm, starting on the day of surgery [4-8].

\section{Conclusions}

A final assessment of the influence of the PIF on the sLC rate is impossible based on the available studies. The differences in the methodological quality of the available studies did not allow for an aggregated meta-analysis involving a joint inclusion of data with direct and indirect evidence. The results of the meta-analysis of the retrospective studies show a reduction of $77 \%$, with a very wide confidence interval and considerable inhomogeneity of the studies. The only prospective-controlled study could not find a significant group difference in the sLC rate, so that the results of further studies with direct evidence are to be awaited.

\section{Acknowledgement}

We would like to thank Gjoko Stojanoski, Department of Urology, St. Elisabeth-Hospital Straubing, Brothers of Mercy Hospital, Straubing, Germany, for his outstanding contribution to the Pianoforte study.

\section{Conflict of Interest Statement}

The authors have no conflicts of interest with the current analysis to declare. No author has direct or indirect commercial financial incentives associated with publishing the article.

\section{Funding Sources}

The authors did not receive any funding.

\section{Author Contributions}

The authors M. May and S. Deutsch were mainly responsible for the article and graphic design, acquisition of articles, analysis and interpretation of the results, and writing the text. B. Hadaschik and S. Lebentrau contributed to the article through continuing revision and critical discussion of the results. For approval and discussion, B. Ubrig and M. Burger finally checked the text.

\section{References}

1 Dell'Oglio P, Mottrie A, Mazzone E. Robotassisted radical prostatectomy vs. open radical prostatectomy: latest evidences on perioperative, functional and oncological outcomes. Curr Opin Urol. 2020;30(1):73-8.

2 Antonelli A, Palumbo C, Noale M, Porreca A, Maggi S, Simeone C, et al. Impact of surgical approach on patient-reported outcomes after radical prostatectomy: a propensity scoreweighted analysis from a multicenter, prospective, observational study (rhe pros-IT CNR study). Urol Int. 2019;103(1):8-18.

3 Kamel MG, Istanbuly S, Abd-Elhay FA, Mohamed MYF, Huu-Hoai L, Sadik M, et al. Examined and positive lymph node counts are associated with mortality in prostate cancer: a population-based analysis. Urol Int. 2020; 104(9-10):699-709.
4 Lebeis C, Canes D, Sorcini A, Moinzadeh A. Novel technique prevents lymphoceles after transperitoneal robotic-assisted pelvic lymph node dissection: peritoneal flap interposition. Urology. 2015;85(6):1505-9.

5 Dal Moro F, Zattoni F. P.L.E.A.T.-Preventing lymphocele ensuring absorption transperitoneally: a robotic technique. Urology. 2017; 110:244-7.

6 Stolzenburg JU, Arthanareeswaran VKA, Dietel A, Franz T, Liatsikos E, Kyriazis, et al. Four-point peritoneal flap fixation in preventing lymphocele formation following radical prostatectomy. Eur Urol Oncol. 2018; 1(5):443-8.
7 Boğa MS, Sönmez MG, Karamık K, Yılmaz K, Savaş M, Ateș M. The effect of peritoneal reapproximation on lymphocele formation in transperitoneal robot-assisted radical prostatectomy and extended pelvic lymphadenectomy. Turk J Urol. 2020;46(6):460-7.

8 Lee M, Lee Z, Eun DD. Utilization of a peritoneal interposition flap to prevent symptomatic lymphoceles after robotic radical prostatectomy and bilateral pelvic lymph node dissection. J Endourol. 2020 Aug;34(8):821-7.

9 Bründl J, Lenart S, Stojanoski G, Gilfrich C, Rosenhammer B, Stolzlechner M, et al. Peritoneal flap in robot-assisted radical prostatectomy: results of a multicenter, randomized, single-blind study (PIANOFORTE) of the efficacy in reducing postoperative lymphocele. Dtsch Arztebl Int. 2020;117:243-50. 
10 Motterle G, Morlacco A, Zanovello N, Ahmed ME, Zattoni F, Karnes RJ, et al. Surgical strategies for lymphocele prevention in minimally invasive radical prostatectomy and lymph node dissection: a systematic review. J Endourol. 2020;34(2):113-20.

11 Lenart S, May M, Burger M, Bründl J. Re: utilization of a peritoneal interposition flap to prevent symptomatic lymphoceles after robotic radical prostatectomy and bilateral pelvic lymph node dissection by Lee et al. J Endourol. 2020;34(12):1276-7.

12 Moher D, Shamseer L, Clarke M, Ghersi D, Liberati A, Petticrew M, et al. Preferred reporting items for systematic review and metaanalysis protocols (PRISMA-P) 2015 statement. Syst Rev. 2015;4(1):1.

13 Quigley JM, Thompson JC, Halfpenny NJ, Scott DA. Critical appraisal of nonrandomized studies: a review of recommended and commonly used tools. J Eval Clin Pract. 2019 Feb;25(1):44-52.
14 Ploussard G, Briganti A, de la Taille A, Haese A, Heidenreich A, Menon M, et al. Pelvic lymph node dissection during robot-assisted radical prostatectomy: efficacy, limitations, and complications-a systematic review of the literature. Eur Urol. 2014;65(1):7-16.

15 Grande P, Di Pierro GB, Mordasini L, Ferrari $\mathrm{M}$, Würnschimmel C, Danuser $\mathrm{H}$, et al. Prospective randomized trial comparing titanium clips to bipolar coagulation in sealing lymphatic vessels during pelvic lymph node dissection at the time of robot-assisted radical prostatectomy. Eur Urol. 2017;71(2):155-8.

16 Seetharam Bhat KR, Onol F, Rogers T, Ganapathi HP, Moschovas M, Roof S, et al. Can we predict who will need lymphocele drainage following robot assisted laparoscopic prostatectomy (RALP)? J Robot Surg. 2020; 14(3):439-45.
17 Sforza S, Tellini R, Grosso AA, Zaccaro C, Viola L, Di Maida F, et al. Can we predict the development of symptomatic lymphocele following robot-assisted radical prostatectomy and lymph node dissection? Results from a tertiary referral Centre. Scand J Urol. 2020; 54(4):328-33.

18 Andrews JR, Sobol I, Frank I, Gettman MT, Thompson RH, Karnes RJ, et al. Treatment outcomes in patients with symptomatic lymphoceles following radical prostatectomy depend upon size and presence of infection. Urology. 2020 Sep;143:181-5.

19 Keskin MS, Argun ÖB, Öbek C, Tufek I, Tuna MB, Mourmouris $\mathrm{P}$, et al. The incidence and sequela of lymphocele formation after robot-assisted extended pelvic lymph node dissection. BJU Int. 2016 Jul;118(1): 127-31. 